\title{
Investigating Autistic Children's Attitudes Towards Strangers with the Theatrical Robot - A New Experimental Paradigm in Human-Robot Interaction Studies
}

\author{
Ben Robins *, Kerstin Dautenhahn*, Janek Dubowski** \\ * Adaptive Systems Research Group, University of Hertfordshire, UK \\ ** School of Psychology 65 Therapeutic Studies, University of Surrey Roehampton, UK
}

\begin{abstract}
In this paper, as part of a larger study into the possible use of robots in therapy or education of children with autism, we studied the effects of two different robot appearances on autistic children's behaviour towards the robot. We used a novel experimental paradigm, the Theatrical Robot, which is discussed in the context of other evaluation methods used in the field of Human-Robot Interaction (HRI). The two appearances used were a plain/robotic and an 'ordinary human' appearance. The response of children with autism towards the plain/robotic robot was notably more social and pro-active. The ordinary-human appearance resulted in avoidance behaviour or 'aloofness', a typical behaviour that autistic children show towards strangers. Implications of these results for our work on robots and autism, as well as other HRI research are discussed.
\end{abstract}

\section{Introduction}

Investigating the use of robots in education and therapy is an active area of research. One interesting application area that we study in the Aurora project (www.aurora-project.com) is to develop robotic toys that might be used for therapy or education of children with autism [5]. We run trials where the children play with robots that can engage them in simple interaction activities, such as imitation and turn-taking games. Contrary to peoples' social behaviour, which can be very subtle and widely unpredictable, the use of robots in the Aurora project allows for a simplified, safe, predictable and reliable environment where the complexity of interaction can be controlled and gradually increased. As part of a longitudinal study with a small humanoid doll [13] we investigated the effects of a robot's appearance on children's behaviour, presenting the robot in a 'plain/robotic' dress as well as in a 'pretty-girl doll' dress. In the current study we pursued a new approach and we utilized a life-size 'robot', a Theatrical Robot. In the following we explain this new experimental paradigm and why we believe that it might also be fruitful in other areas of HRI research.

\section{Evaluation of Human-Robot Interac- tion}

Robot-Human Interaction research has benefited from the availability of sophisticated human-size robotic platforms such as Asimo (Honda). Humansized robots can afford interactions apparently at "eye level". Increasingly researchers are investigating how people respond to different appearances or behaviours of robots in order to inform further robot development. Such studies closely resemble those in psychology and social sciences into human behaviour. However, conducting and evaluating user studies that meet the requirements and standards of human-human interaction studies is still a big challenge. In humanhuman interaction studies, methods often involve the naive (test-) subject, in addition to a trained and preinstructed person who, unknown to the test subject proper, exhibits behaviours related to different experimental conditions, see study by [11] which involved a confederate experimenter as one of the apparent subjects.

Can a robot be a confederate experimenter? It implies that the robot needs to be equipped with several non-trivial behaviours that support interactions and communication with humans, coping with the whole range of erratic, idiosyncratic, or otherwise unusual behaviour that humans are likely to perform in any such studies. Such requirements pose big technological challenges, i.e. they demand sophisticated programming and engineering skills, and the development process will take an extended period of time. Compared to instructing a human in human-human interaction studies the effort to realize user studies with robots is immense and poses significant obstacles to the advancement of research in the field. 


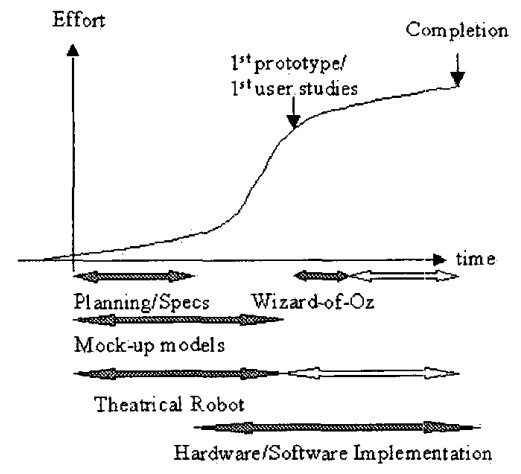

Figure 1: Sketch of a typical development time line of socially interactive robots

The Wizard-of-Oz technique, as a rapid prototyping method, is a widely used evaluation technique in HCI and HRI research (e.g. [10]) that can result in proof-of-concept evaluation data with a prototype version. It involves a human who is (unknown to the test subjects) controlling the behaviour of the system, ranging from full teleoperation to partial control of 'higher level' decision making processes. However, if the human "wizard" faces a complex behaviour arbitration problem then the cognitive load on the human can be substantial. In order to address some of the issues mentioned above, we propose an alternative paradigm that provides help in evaluating human interactions by using a Theatrical Robot, a life-size, embodied, simulated robot which allows to investigate requirements of robot design even prior to any hardware and software development. This new paradigm might complement already existing approaches and can be applied most usefully in very early phases of the robot design. The Theatrical Robot consists of a human instructed to behave and/or appear like a robot. The human should be a professional or person trained to perform pre-scripted behaviours, as needed for experimental protocols, reliably and with high precision.

Figure 1 shows a sketch of a typical development time line of socially interactive robots. During the initial planning phase mock-up models might be used [2]. Once a system's main components have been implemented, a Wizard-of-Oz study is applicable. As soon as working prototypes exist user studies can be conducted. The proposed Theatrical Robot allows to conduct user studies even from the early phase of planning and specification onwards throughout the whole development of the robot. Once working prototypes exist they are likely to become less useful since user studies can be run with the "real system" rather than its embodied simulation.

In our present study we used the Theatrical Robot to evaluate the effects of robot appearances on children with autism in the context of therapy and education.

\section{Robots and Children with Autism}

Autism is a spectrum disorder which comprises a large range of different abilities and skills [12]. All children with autism show impairments in the following areas: social interaction, communication, and imagination. Amongst the many theories put forward to explain the nature of the three deficits associated with autism is the Theory of Mind Hypothesis. The ability to 'mentalize', or appreciate the mental states of others, is evident in typically developing children around the fourth year of life, while it does not seem fully developed in children with autism, e.g. [1]. For the purpose of this paper it is important to note that people with autism show difficulties in interpreting other people as people.

Children with autism show a poor use of eye contact, as an inability to learn 'the language of the eyes', that is, to learn to use and understand the signals associated with particular mental states. An autistic child will insist on sameness, expressing a need for pattern and structure that frequently exhibits itself in repetitive play which is very common and can take many forms. One account of why such patterns of behaviour develop and persist with these children could be attributed to the sensory side of the condition $[17,8]$. For some children, the need to maintain sameness is very strong in order to moderate potentially overpowering sensory stimulus. This might extend to the general environment and any change or movement of furniture in a room, for example, can cause distress. In many cases, what applies to objects also applies to other people who are often regarded as if they are just objects. Peter Hobson [9] studied the behaviour of children with autism when greeted by a stranger and found that they did not seem to react with feelings to his presence and his orientation towards themselves. The children with autism did not seem interested in the stranger and often didn't even look at him. Thus, perhaps the most complicated and unpredictable thing that an autistic person might encounter is another person, unfamiliar to them. As a result, it is not just that the children might demonstrate a preference to interacting with objects rather than with other people, but, as Hobson [9] suggests, children with autism often seem to relate to a person as an object. 


\section{Current work}

If some children with autism demonstrate a preference to interact with objects rather than people how would they interact with a humanoid robot? This paper reports findings focusing on aspects of the robot's design that can facilitate the interactions of children with autism with the robot. Earlier work reported that the children preferred simple designs and a predictable environment in their interaction with toys, and that they approached social objects (they used various types of dolls) more readily if they were simple in appearance [6]. In our investigation into the effects of the robot's design, we conducted two studies: A study with a Theatrical Robot and a study with a small humanoid robotic doll. The present paper focuses on the study with the theatrical robot, the other results are published in [15].

\subsection{A study with a life size 'robot'}

In our current and previous studies we used a small humanoid robot with a pretty, detailed face and girl's clothing [13]. Bearing in mind the social interaction impairment of autistic children, and their reaction to other unfamiliar people, or 'strangers', one of the questions posed by the current research is how children with autism may react to a life size robot with a full range of possible interactive movement. At present, controlling a humanoid robot with many degrees of freedom requires state-of-the-art computing and engineering skills, which lies outside the scope of a project in assistive technology. Full-sized humanoid robotic platforms are also highly expensive and safety issues are a main concern.

In order to address this lack of the availability of a safe, easy to control full-sized humanoid robot that can be used in our studies we developed a novel approach by using a Theatrical Robot - a mime artist a person who was dressed and acted like a robot (see figure below).
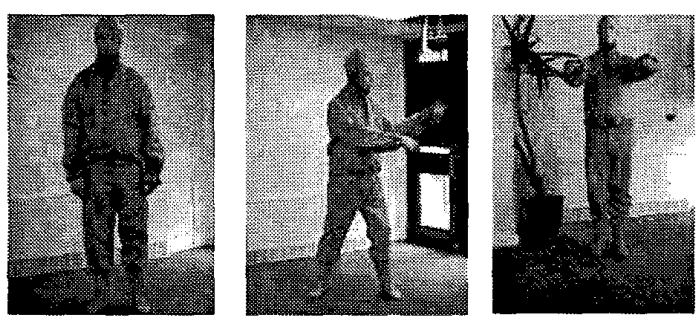

Figure 2: The theatrical 'robot' in its various interaction modes

In this study, we compared the children's level of interaction with and response to the mime artist in two different scenarios, one when he was dressed like a robot, and the other when he was dressed as an ordinary human. The trials with the two scenarios took place on the same day, approximately one hour apart. The set-up of the trials in both scenarios were identical, i.e. they took place in the same room, and the mime artist performed an identical, prescripted repertoire of movements in both cases, closely mimicking the movements of the small humanoid robot. This whole study with the mime artist performing in two scenarios was repeated again for the second time, two month later, with very similar results.

\subsection{The Research Question}

Children with autism have been found to not react socially to strangers, they rather tend to exhibit avoidance and indifference. However, they appear to respond positively to the simplified environments provided by computer systems and robots. Thus, we hypothesize that the children would react (socially) more proactively towards the mime artist in its "robotic" appearance (the plain version) as opposed to the "natural" human-like appearance including e.g. full facial features.

\section{The Trials}

The trials took place in a primary school in Essex, UK, and have been designed to allow the children to have unconstrained interaction with the mime artist with a high degree of freedom, on their terms to begin with (providing it is safe for the child and safe for the robot), and to build a foundation for further possible interactions with peers and adults using the robot as a mediator $[16,14]$. Four autistic children age 5-10 participate in the trials that have previously been diagnosed with autism. Each child participated in four trials with the theatrical 'robot': both appearances were presented on two different days (trials 1\&2). The trials were conducted in a familiar room often used by the children for various activities. The room size is approx. $4.5 \mathrm{~m} \times 3 \mathrm{~m}$, with a carpeted floor.

\subsection{Quantitative Analysis}

The video data from each and every trial for a given child was segmented into one second intervals. The trials were coded by scoring three elementary behaviours: Eye Gaze (when directed at the robot), touch (when the child touched any part of the robot) and near (this included the child approaching the robot and staying in close proximity to the robot regardless of the child's other behaviours). The coded data for each trial was then summed up and yielded the total number of occurrences of each behaviour during a specific trial and 
the total duration the child was engaged in each behaviour during that trial. As some of the trials varied in duration, the total duration of a behaviour was transformed to a proportional representation of the duration of behaviour relative to the duration of that specific trial.

\subsection{The Theatrical Robot}

The mime artist who performed the Theatrical Robot role was a white male, $175 \mathrm{~cm}$ tall with average build. The 'robotic' costume included a complete head cover, mask, shirt, gloves, trousers, socks and shoes - all painted in the same light gray colour (see below). The ordinary human costume included brown shoes, dark trousers, an open brown jacket, and a light colourful shirt. The mime artist's movement repertoire progressed from stillness, through simple robotic movements to more human like interactional gestures, including the simple robotic movements similar to the movements of the humanoid robotic doll (i.e. arms up and down, legs up down, head side to side). The mime artist performed the same movement repertoire in the same order in all the trials (including the trials where he was an ordinary person- a stranger). The mime artist was not responding to the children, during the whole trials his eye gaze was direct straight forward.

Before each trial, the mime artist was standing still in the far end of the room, ready to start his movement repertoire as soon as the child entered the room. The investigator was located behind a set of curtains, at the other end of the room, operating one of the cameras. He wasn't visible to anyone in the room. The other camera, operated by remote control, was set to 'standby' mode ready to record. The children were brought to the room by their carer, one at a time. The carer, staying near the entrance, did not intervene in the trial procedures, nor did she give any instruction to the child except for the initial drawing of the child's attention to the mime artist if it was needed. The child then, was left to observe and interact with the mime artist, should he choose to do so. The mime artist continuously performed his repertoire of movements, which included approximately one minute of stillness, two minutes of simple robotic movernents and a further two minutes of human gestures. Figure 7 shows interaction during these variuos types of movements. The trial, lasting approx. five minutes, stopped at the end of this sequence of movements.

\subsection{Results}

The graphs below compare the response of all the children to the two appearances of the mime artist (robot and human) in terms of Touch, Gaze and Near as observed in two sets of trials (Figures $3,4,5$ )

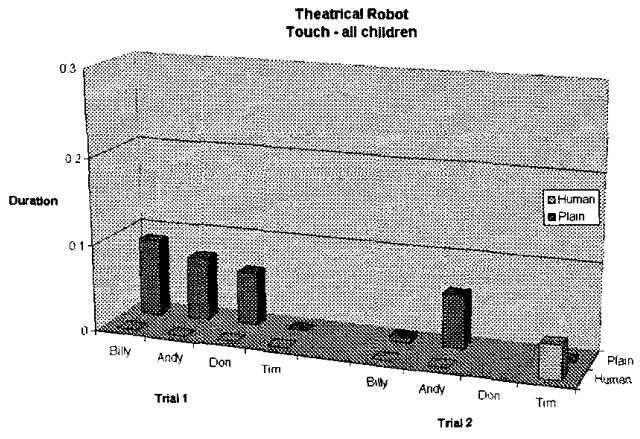

Figure 3: Scores for the behavioural criteria of Touch

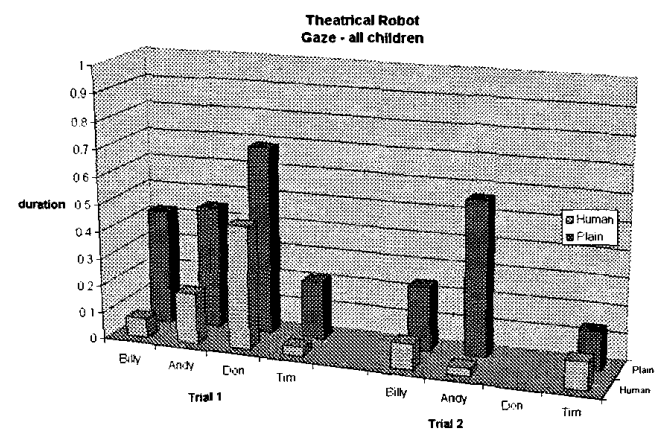

Figure 4: Scores for the behavioural criteria of Gaze

The graphs show a remarkable difference in the amount of time the children interact with the mime artist when he appeared in his Theatrical Robot costume, and when he appeared as an ordinary human. All children showed notably increased levels of interaction in the behavioural criteria of Gaze, Touch \& Near, when the mime artist appeared as a theatrical 'robot'. Moreover, the second set of trials that took place two month later (trial 2 in the graphs) shows very similar results.

The observation of the video recordings of the trials with the mime artist showed a striking difference in the children's reactions towards the mime artist when he was in his Theatrical Robot costume and when he was wearing an ordinary person's clothes. Note, that the trials took place with the same children and in the room with the same experimental settings. When the mime artist presented himself as an ordinary person a stranger, he was being avoided or ignored. Typically, children maintained distance to the mime artist and eye contact was avoided. What is sometimes described as 'aloofness' but is a form of avoidance behaviour, can 


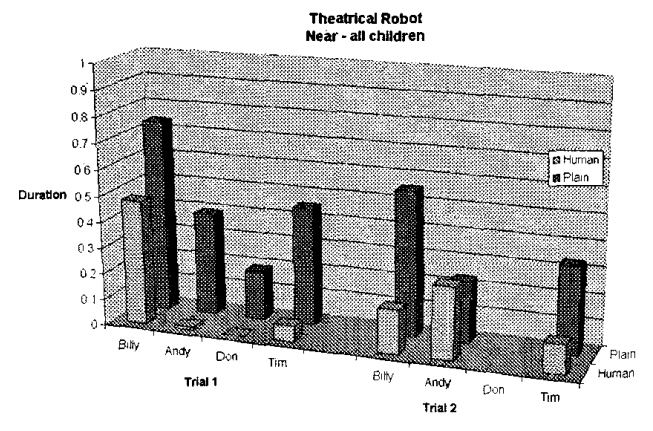

Figure 5: Scores for the behavioural criteria of Near

be observed in figure 6 .
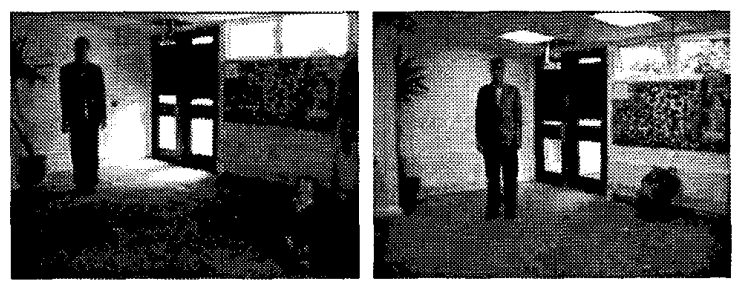

Figure 6: Avoidance behaviour towards the mime artist when dressed as a human

In the trials with the Theatrical Robot, as soon as the 'robot' is noticed, he, (it) is approached by the child who in most cases immediately makes physical contact with it as can be seen in figure 7 . The child's attention is maintained and when the Theatrical Robot begins to go through his 'robotic movements' the child becomes even more bold in his interaction, e.g. the child begins to mimic the robot's movements and even maintains physical contact with the 'robot' as it is moving (see below).
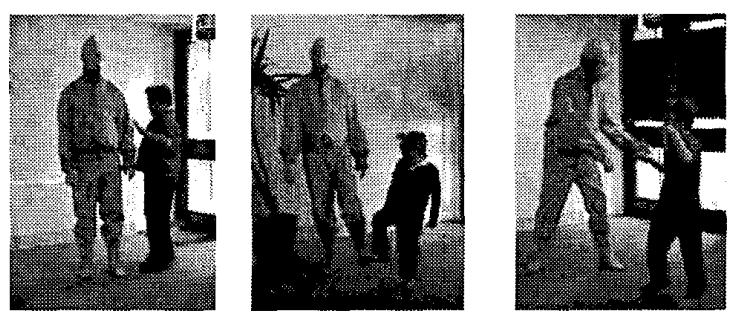

Figure 7: (from left to right) Andy Billy and Chris making physical contact with the theatrical 'robot'
The pictures below derive from the repeated trial that took place two months later. Here we can see the same child, Andy, reacting in very similar way. As soon as Andy noticed the 'robot', he approached it and made physical contact. Moreover, we can see that Andy's gaze during this interaction is often being directed to the robot's face.
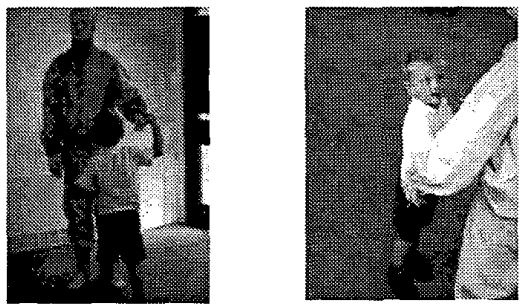

Figure 8: Andy in a repeated trial two months later.

\subsection{Discussion of Results}

All four children with autism tested showed notable differences in how they interacted with the mime artist in the two experimental conditions: as a Theatrical Robot (robotic, plain appearance), or with ordinary human appearance. This confirms our initial hypothesis which originated in research where people with autism interact with other people. Note, that the study comprised four children which were exposed to the two conditions only twice. Possibly, after repeated exposure to the children the mime artist in his ordinary human appearance will no longer be a stranger, but become a familiar person. Similarly, experimenters who work regularly with the same children become over time more and more familiar to a child who is then likely to change behaviour towards that person. However, in this case the experimenter or therapist can develop a meaningful "relationship" to the children, which is very different from what can be expected of a robot. Further in-depth studies into the role of robots and "strangers" in the therapy and education of children with autism might shed more light on these issues and provide additional experimental evidence. However, the results at present are nevertheless striking in showing notable differences in the two experimental conditions studied. An important implication of our findings for the use of robots in therapy and education of children with autism relates to the question of whether one should use humanoid robots that closely resemble human beings (e.g. possessing a lot of facial features such as eyes, month, eye brows etc.), as suggested e.g. by [3], or rather utilize machine-like, clearly non-humanoid robots, as ar- 
gued e.g. in [4]. The preliminary evidence presented in this paper clearly supports the case of using simple robots with few features. However, once a robot becomes familiar, it might be possible to gradually change the appearance towards more human-like appearance, which could also assist the children in generalising experiences from interactions with robots to interactions with people, in line with the analysis presented by Ferrara and Hill in their studies with different toys for children with autism [6].

\section{Conclusion}

In this paper we presented a case study in robothuman interaction which investigated the influence of different appearances of a robot on children with autism. We used a novel experimental paradigm where a professional mime artist "simulated" the appearances and behaviours of a robot. Instructing the mime artist and providing different clothings suitable for the experiments was highly efficient in terms of time and computational efforts, as compared to conducting the same study using a human-size humanoid robot. We show that the theatrical robot can provide experimental results on user studies even before any prototypes of robotic systems have been built and before a Wizard-of-Oz study can be conducted. Given the effort it takes to design and redesign the appearance of robots, we believe that our approach could offer significant advantages. We believe that the Theatrical Robot approach can complement already existing approaches to user studies in robot-human interaction, such as mock-up models or the Wizard-of $-\mathrm{Oz}$ technique, in early stages of robot development before design decisions have been finalised. The field of robot-human interaction is still in its early days, so any method that allows to explore the design spaces of socially interactive robots (cf. [7]) could bring us closer to the goal of robots that can be truly useful in various application areas ranging from service robotics to the use of robots in therapy and education of children with autism.

\section{References}

[1] S. Baron-Cohen, A. M. Leslie, and U. Frith. Does the autistic child have a "theory of rnind". Cognition, $21: 37-46,1985$.

[2] C. Bartneck and J. Hu. Rapid prototyping for interactive robots. In Proc. IAS-8, pages 136-145, 2004.

[3] C. Breazeal and A. Foerst. Schmoozing with robots: Exploring the boundary of the original wireless network. In Proc. CT 1999, pages 375-390, 2001.

[4] K. Dautenhahn. Robots as social actors: Aurora and the case of autism. In Proc. CT99, pages 359-374, 1999.

[5] K. Dautenhahn and I. Werry. Towards interactive robots in autism therapy: Background, motivation and challenges. Pragmatics and Cognition, 12(1):135,2004

[6] C. Ferrara and S. D. Hill. The responsiveness of autistic children to the predictability of social and nonsocial toys. Autism and Developmental Disorders, 10(1):51-57, 1980.

[7] T. Fong, I. Nourbakhsh, and K. Dautenhahn. A survey of socially interactive robots. Robotics and $\mathrm{Au}$ tonomous Systems, 42(3-4):143-166, 2003.

[8] G. Gillingham. Autism: Handle with Care: Understanding and Managing Behaviour of Children and Adults with Autism. TX. Future Education Inc, Arlington, 1995.

[9] P. Hobson. The Cradle of Thought. Macmillan, London, 2002.

[10] H. Hüttenrauch, A. Green, M. Norman, L. Oestreicher, and K. S. Eklundh. Involving users in the design of a mobile office robot. IEEE Transactions of Systems, Man and Cybernetics, 2004.

[11] H. L. Roediger III, M. L. Meade, and E. T. Bergman. Social contagion of memory. Psychonomic Bulletin and Review, 2003.

[12] R. Jordan. Autistic Spectrum Disorders - An Introductory Handbook for Practitioners. David Fulton Publishers, London, 1999.

[13] B. Robins, K. Dautenhahn, R. te Boekhorst, and A. Billard. Effects of repeated exposure of a humanoid robot on children with autism. In S. Keates et al., editor, Designing a More Inclusive World, pages 225236. Springer Verlag, London, 2004.

[14] B. Robins, P. Dickerson, P. Stribling, and K. Dautenhahn. Robot-mediated joint attention in children with autism: A case study in a robot-human interaction. Interaction studies: Social Behaviour and Communication in Biological and Artificial Systems, John Benjamins Publishing Company, Amsterdam, 5(2) in press.

[15] B. Robins, K. Dutenhahn, R. te Boekhorst, and A. Billard. Robots as assistive technology - does appearance matter? Submitted to the 13th IEEE International Workshop on Robot and Human Interactive Communication - RO-MAN , Kurashiki, Japan, 20-22 September 2004.

[16] I. Werry, K. Dautenhahn, B. Ogden, and W. Harwin. Can social interaction skills be taught by a social agent? the role of a robotic mediator in autism therapy. In M. Beynon et al., editor, Proc. CT2001, LNAI 2117, pages 57-74, Berlin Heidelberg, 2001. SpringerVerlag.

[17] D. Williams. Autism: an Inside-Out Approach. Jessica Kingsley, London, 1996. 